\title{
Observaciones con Pentotal \ Curare en maternidad
}

\section{Por el doctor JOAQUIN PRADO VILLAMIL}

El Pentotal es un agente anestésico. - más bien hipno-analgésico de la serie de los barbitúricos, cuyo nombre quimico es el Etil-metil-butil-Tio-barbiturato- de Sodio; por contener en su composición química una molécula de azufre goza de la propiedad de ser menos tóxico y más potente que sus congéneres. En el comercio se encuentra en forma de polvo amarillento en ampolletas de 0.50 gramos 0 de un gramo, para diluír en agua bidestilada en el momento de usario.

En anestesiología se usa de diversos modos, todos a cual más útil y eficcz. Sirve para inducir la anestesia geno: Al con la característica de provocar paulatinamente un sueño agradable. muy parecido al natural, sin excitacio nes, de tal manera que el paciente pasa de despierto al primer plano de anestesia quirúrgica, sin darse cuenta de ello, sin que le quede un recuerdo desagradable de la experiencia a que tue sometido, sin sensactón de so focación nf angustia. Una vez llegado a este plano, ya se puede continuar con el anestésico inhalado escogido sin molestia alguna. Para este caso se requioren de 0.25 a 0.40 gramos administrados por vía venosa en una solución acuosa al $2 \% 0.21 \% \%$.

También se usa como anestésico único en una buena contidad de in- tervenciones quirúrgicas. Para tal efecto lo empleamos simultáneamente en concentraciones distintas: una de ellas es al $2 \%$, o sea la misma que ya mencionamos para obtener la inducción anestésica y que nos va a permitir administrarlo además como dosis fuerte para reforzar la acción cuando el paciente trata de superficializarse; la otra concentración que usamos al mismo tiempo y por la misma aquja, es al 2 por 1.000 gota a gota en suero dextrosado y es la encargada de mantener suaremente el plano basal anestésico. Con este método se locra obtener anestesias de -varias horas de duración que combinadas adecuadamepnte con drogas auxiliares (Curare, etc.), permiten electuar intervericiones de alta cirugía.

Por vid intramuscular al $2 \%$ tiene su aplicación para lograr la inducción y aun anestesia en niños de muy corta edad, siendo posible practicar en

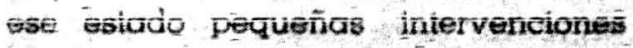
quirúrgicas. Este método está en experimentación muy satisfactoria.

Como lefe del Departamento de Anestesia del Hospital Militar de Bogo tá desde el año de 1943, he tenido oportunidad de usar el Pentotal en todas sus variedades de administra. ción en un poco más de 4.000 - casos, sin haber tenido jamás ninguna com- 
piicación seria. Es una droga-que mientras más se le usa, más conficmza Y cariño se le toma por los incalculables servicios que presta.

Por esta razón. cuando aparecieron sus primeras indicaciones en Obstetr. cic lo ensayé con entusiusmo y cot buen exito en unas 60 pacientes, pero desalortunadamente no llevé en ese tiempo un estricto- "record" de esos casos, razón por la cual no los incluyo en el presente trabajo.

Son muchos $y$-muy interesantes los estudios que se han efectuado con el Pentotal en el campo de la obstetricia pecando algunos de los que he traido la oportunidad de leer de pasar. se de aptimistas. Y sor también muchas las opiniones verbales de algu. nos colegas en contra de la droga y la miran a veces con terror, ya sea porque han tenido algún tracaso en su empleo o ya por haberse formado fal. sa opinión de su uso al oír comentarios poco cutorizados.

Fueron estos los motivos que me movieron para estudiar el Pentotal en maternidad de una manera más estricta $y$ objetiva y poderme formar Is: una opinión más de acuerdo con la realidad. Las observaciones las lle. vé a eabo en su gran mayoría en la Clinica Primero de Mayo y unas pocas en la Clínica Santa Rosa de esta ciucad Lo que puảe observar en cuanto se reflere a la acción del Pentotal. sobre el trabajo del parto y sobre los efectos que puede causar sobre el niño, están acordes en general con to encontradio on otros estudios muy se. rios hechos sobre una estadistica muy grande, como ocurre por elemplo con el trabajo del doctor lorge Henao P., as Medellín, quion lo experimentó en 2.800 pacientes.
En mi caso particular me propuse observar cuál es el efecto de cada dosis sobre el feto, sobre la frecuencia de las contracciones uterinas, sobre la du. ración y calidad de ellas, qué ocurria on las maniobras obstétricas llevadas a cabo baio anestesia con Pentotal etc., etc. como purde verse con deta. lle en los cuadros que adiunto. Asi mismo aproveché la ocasión para observar la manera de actuar el Curare en los perinés contracturadós y a su debido tiempo-comentaré el asunto.

En todos los casos úsé el Pentotal por víc intraveriosa en solución acuosa al $2 \frac{1}{2} \%$; esta concentración es muy cómoda porque en todas partes se encuentran jeringas de 20 c. c. y las ampolletas de la droga más comunes y corrientes son de 0.50 gramos que diluyéndolo en 20 c. c. de agua nos da la solución deseada. Las soluciones al $2 \%$ son igualmente buenas.

Las técnicas de administración endovenosa del- Pentotal, que experi. menté fueron especialmente dos, a saber: a) Administración directa, y b) Administración combinada con suero. Veámoslas:

a) Administración directa. Una vez hecha la solución y debidamente contenida en la jeringa de 20 c. c., se hace la punción venosa con unc aguia nú nero $22 y$ se vo inyoctando lo droga directamente en el torrente circulato rio de acuerdo con las normas que adelante diremos. Como la aguja $y$ jeringa se deben mantener en su sitio durante todo el tiempo requerido, es muy cómodo no usar la jeringa directamente empatada a la acuja, sino conectarlas por intermedio de un tubo de corucho de muy pequeño calibre $y$ de unos 50 Ctms. de largo, ad las su 
cestvas dosis se facilitan enormemen. te. Este es el método empleado cuando el desprendimiento de la presentación es inminénte y se calcula que ocurrirá dentro de breves minutos.

b) Administración combinada con suero. En este caso se practica previa mento und venoclisis con 500 c. c. de suero dextrosado al $5 \%$ y por intermedio de una llave de tres vías, o en su defecto puncionando el caucho del suero con la aguja proveniente de la jeringa y caucho del. Pentotal al $2 \frac{1}{2} \%$, se comunican los dos sistemas. Esté método tiene la ventaja de mantener siempre permeable la aguja de la vena. Las dosis de Pentotal que se van necesitando en. tran mezcladas con el suero. Su uso se impone cuando se calcula que el parto se efectuará en un tiempo más - menos largo: tiene rambién la ventaja de mantener a la paciente bien hidratada $y$ en mejores condiciones generales.

En cuadro adjunto inserto las observaciones personales que llevé a cabo con solución de Pentotal al $2 \frac{1}{2} \%$ por via venosa; para luégo cómentarlas y sacar las deducciones del caso.

Del estudio de los presentes cua dros vemos los siquientes hechos que analizaremos: primero desde el punto de vista materno y segundo desde el punto de vista fetal.

19 Consideraciones reterentes a la madre. Usamos el Pentothal en el periodo final del parto, con cuello completamente dilatado o muy próximo a 3u dilatación completa, por lo general con presentación ya encajada.

El plano anestésico no lo profundi. zamos sino en caso de intervencionee obstetricas, de lo contratio se mantio. ne a la paciente únicamente en grado de inconsciencia, pero conservando los movimientos reflejos; no es capaz de contestar a ninguna pregunta, pues es. tá sumida en profundo sueño, pero cuando viene la contracción uterina irata de pujar ligeramente, se apoya on las manos para hacer esfuerzo, le vanta la cabeza y se quela; pasada la contracción uterina continúa dormida cgradablemente.

Con excepción de una paciente muy nerviosa y excitada, todas entraron paudatina y agradablemente on un sueño muy semejante al fisiológico. En todos los casos la calma y la tranquilidad siguieron a la inyección de Pentotal, nunca observamos exageración algunc de la excitación.

Sistemáticamente les averigüé a to das las entermas al dia siguiente si se habian dado cuenta del nacimiento de la criatura y todas estuvieron de acuerdo en que no recordaban absolu. tamente nada desde el momento de la inyección de Pentotal hasta mucho rato después del parto. La mayoría recobraron la consciencia cuando yo habian sido trasladadas a su carna.

El procedimiento para inyectar el Pentolal en términos generales es el siguiente, sea que empleemos el mó todo directo o el combinado con suero dextrosado: En el momento que se decida comenzar la analgesia, se inyecta lentamente la solución de Pentotal. haciendo que la enferma cuente en voz alta de uno en adelante: llega un momento (al coniar entre 17 y 25) que la palabra se torna dificll y como pastosa a los pocos segundos sigue con dificultad repitiendo el mismo núme. ro "o trata de continuar contando con gran trabajo a instancias del anesto 
sista, poco despues ya no es capaz de hablar. En este momento (por lo ge nèral, se han gastado de 0.14 a 0.20 gramos) se debe suspender_la inyección y observar a la enferma, si trata de superficializarse se inyectan unos 0.03 a 0.05 yramos más. Debe vigilar. se cuidadosamente la respiración de la enferma que en todo momento tie ne que ser libre y regular, si es el caso se hace tracción del maxilar inferior para facilitar el paso del aire. En los minutos siguientes se inyectan de 0.03 a 0.10 gramos de Pentotal-cada vez que venga la coñtracción uterina, graduando la dosis de acuerdo con las nocesidades.

En cuanto a la facilidad para anesteciar a las pacientes podemos dividirlas en tres grupos: a) Las sensibles que se duermen con muy poca cantidad de la droga, como ia de la observación número 23, que con 0.07 gra. mos entró en sueño, este grupo es el ideal para la anestesia con Pentotal: b) Las normales que necesitán entre 0.15 y 0.25 gramos, son las más numerosas; c) Las resistentes que requieren dosis altas y a veces, sin embargo. no son satisfactorios los resultados; éstas son malas pacientes para esta clase de anestesia.

Entremos ahora a clasificar los dis. tintos aspectos interesantes en cuan. to se refieren a la madre.

Pasidad. Usamos la anestesia indis. tintamente en primíparas y multiparas, sin encontrar mayor diferencia entre unas $y$ otras, asi:

Primipcras .. ......... 10 casos

Segundo embarazo ....... 2 casos

Tercer embarazo ........ 8 casos

Cuarto embarazo . . . . 2 casos
Quinto embarczo ....... 4 cabus

Sexto embarczo ........ 2 casos

Séptimo embarazo ........ 1 caso

Décimo embarcizo ...... 1 caso

Edad embarazo. El Pentotal no debe usarse como analgésico obstétrico sino en embarazos a término o muy próximos a el, por la depresión resp: ratoria letal que siempre causa, aur. a dosis bajas para la madre. Si lo usamos en los prematuros donde los cen. tros bulbares no han llegado aún a su pleno desarrollo, podríamos carisar una depresión mortal para el teto que no es capaz de recuperarse: Debe, pues, proscribirse su uso de los ocho meses de embarazo para atrás.

Presentación. La mayoría fueron cefálicas. Solamente hubo una podálica en embarazo único, y otra en el segundo feto de un gemelar. Aquí caif hacernos la preginta si el Pentota. es benéfico en las presentaciones podálicas en cuanto por su acción depresora respiratoria aleja un poco la complicación de la respiractón prematura de la criatura cuando-la cabeza última aún no se ha desprendido. Se ría digno de observarse en un buen número de casos. Por demás está decir que en las dos podálicas que se nos presentaron todo marchó normalmente.

Tiempo de trabajo. En nuestros casos contamos con madres que esta. ban en trabajo desde hacía dos ho ras hasta 28 horas. Me dejó la impresión que mientras más horas lleven de-trabajo, el Pentotal obra mejor, por dos razones: primerc, porque la enferma está un tánta agotada y por consiguiente hay menos resistencia física a la acción de la droga segur. 
co, putqu la enterma unela E. ait vio rápido de sus dolores y colabo ra mejor con el anestesista.

\section{Encajamiento de la presentación.} Como la analgesía se lleva a cabo en el final de parto lo común y co riente es que la presentación ya este encaiada. Sin embargo en las gran des multiparas con bolso integra st puede administrar antes de encajarse. En nuestra estadística hubo tres en estas circunistancias.

Membranas. Por lo general es más cómodo romperlas antes de comenzar la anestesia. En las grandes multiparas se puede hacer las dos cosas simultáneamente. En nuestros casos se comenzó la anestesia antes de romper las membranas en nueve casos, y después de efectuada la maniobra en 21 .

Dilatación del cuello. Para iniciai la anestesia debemos esperar que esté en unos seis a ocho centímetros en las multíparas y muy cerca de la dilata ción completa en las primíparas. Esto coń el objeto de no prolongar demasiado la anestesia y evitarle al leto mucho tiempo de depresión.

Dosis de Pentotal La inicial, que es la mayor de todas, es la responsable de los trastornos que ocurren en la mécánica uterina. Las dosis suplemen. tarias no tienen - mayor influencio siempre que sean pequeñas. La mayor dosig inicial requerida no nuestros s:termas fue de 0.36 gramos. La mayor dosis total en el lapso de un parto tue de 0.58 gramos.

Frecuencia de las contracciones. Es tudiando detenidamente cada caso de nuestro cuadro, puede observarse claramente-que: $1^{\circ}$ Cuando se emplean dosis miciales menores de 0.15 gra mos de Pentotal, el ritmo de las contracciones sigue igualmente trecuente a las que temía la paciente antes de la inyección.

$2^{\circ}$ Cuando la dosis fluctúa entre 0.15 y 0.20 gramos, la frecuencia con que se suceden las contracciones su. tre un retardo de un minuto más o menos.

$3^{\circ}$ De 0.20 gramos para arriba el retardo puede llegar a ser de dos a cinco-minutos, es decir, que una paciente que tenía los dolores cada dos minutos, pueden espaciársele a cinco o siete minutos. En resumen, el Pentotal trata de disminuir la frecuencia de las contracciones.

Calidad de la contracción. Observamos que cuando se requieren hasta 0.20 gramos de dosis inicial de Pen total, la ealidad de la contracción uterina es sensiblemente igual. De 0.20 gramos en adelante ésta se hace cada vez menos buena, proporcionalmente a la dosis empleada.

Duración de la contracelón. Sigue muy de cerca a la frecuencia. Hasta 0.15 gramos de dosis inicial, las cosas siguen más o menos íguales. Entre 0.15 y 0.20 gramos la duración de la con tracción se baja en unos cinco segundos. De 0.20 gramos en adelante va disminuyendo la duración a medida que aumenta la dosis. En el caso númeto is habia contracciones que duraban 40 segundos; fue necescrio inyectar 0.32 gramos de Pentotal e inmediatamente la duración de las contracciones uterinas bajó a 15 segundos nada mas; hubo que inyectar $P$. tocín y ayudar con expresión abdomi. nail. 
Alumbramiento. No parece afectar. lo el Pentotal en ninguna forma, como puede verse. Este sobrevino:

A los 3 minutos en 1 paciente

A los 4 minutos en 1 paciente

A los 5 minutos en 8 pacientes

A los 6 minutos en 3 pacientes

A los 7 minutos en 3 pacientes

A los 8 -minutos en 6 pacientes

A los 10 minutos en '2. pacientes

A tos -12 minutos en 2 pacientes

A los 15 minutos en 2 pacientes

A los 40 minutos en -1 paciente

Retención plact. en 1 paciente

Háciendo el cómputo nos da como promedio siete minutos entre el parto $y$ el alumbramiento.

Maniobras obstétricas. Las que en sequida mencionamos fueron llevadas a cabo exclusivamente bajo aneste sid con Pentotal.

\section{Expresión abdominal en cinco} casos para ayudar al desprendimiento.

2 Episiotomías 4. En unos casos por presumirse ruptura inminente del peri. né. En otros para aplicación de forceps.

\section{Fórceps, asi:}

(1) Por deflexion . . . . . . . . . 3

b) Por sufrimiento fetal ....... I

c) Por variedades posteriores ... 2

d) Por procidencia cordón ..... 1

Todas con buen extlo $y$-buena caltdad de analgesia Anotemos de paso que la yersión nunca debe hacerse con Pentotal debido a la falta de relajacion uterina que puede acarrear ruptura de la matriz.

Ocitócicos. Cuando los necesitamos slempre recurrimos al Pitocín (una ampolta diluida en nueve c. c. de sue ro) aplicándoio por uñidades. Es muy buen recurso para- contrarrestar el éfecto depresor de Pentotal sobre la fibra uterina. En nuestros casos lo usamos con este fin nueve veces (entre dos y cuatro unidades) y respondió -satisfactoriamente. Es evidente que con esta anestesia hay mayor trecuen. cia en el uso de los ocitocicos

Colaboración de la enferma. Con el Pentotal como con todos los demás agentes anestésicos, la colaboración que nos presta la enferma al pujar en el momento de la contracción, se dis. minuye notablemente y cún a veces queda abolida. A la fibra uterina le queda todo el trabajo de la expulsión. por eso es una gran ayuda la expre sión abdominal suave.

Complicaciones. Debemos anticipar que no tuvimos ninguna complicación materna como efecto directo del Pentotal. Las dos que acurrieron fueron inherentes al parto, a saber:

a) Desgarros perineales solamente uno, en una aplicación de fórceps. El uso del curare por una parte y la epísiotomía preventiva por "otra, hicieron que los desgarros espontáneos fueran casi nulos.

b) Hemorragia. Hubo un caso serio cuando la enferma ya estaba en su cama, una hora después del parto; se acompañó de shock hemorrágico $y$ sue necesaria una transfusión de sángre total. Es muy dificil conciuir si el Pentotal tomó parté en este accidente, no tenemos bases para juzgarlo, y por otre parte fue muy tardía, cuando la droga ya no actuaba.

No debemós olvidar nunca que es muy frecuente que el Pentotal pro duzce apneas por su efecto depresor sobre el centro respiratorio. Los que 
irancinos con ia anestesic estamos acosiumbrados a verlas en un gran porcentaje de casos, pero teniendo a - La mano un aparato de suministrar oxigeno a presión el accidente reviste la más minima importancia, es una cosa baladi. De ahi la necesidad de tener aparatos adecuados de oxigeno en las salas de partos.

Aprovechamos la ocasion para te cordar que et Pentotal no provoca vo milos, lo que constituye una gran ventaja sobre los otros agentes anestésicos-que casi en su totalidad desenca- denan esta molesta - complicación. En nuestros casos nunca se presentó.

Cuando hay desgarros perineales o episiotomías que suturar, una vez ter: minado el parto se reparan bajo anestesia pentotálica; en este caso se continúa administranco la draga.

$2 \circ$ Contidergetomes ruferontes al folo. Cuando el niño nace es muy tácil comprobar el color, el grado de anes. tesia, la calidad de la respiración, si ésta es inmediata o e demora alovín Liempo, si hay meconio o no, etc. otc. Pero mientras está en la matriz lo úni. co que podemos verificor es el latido del corazón; los demás fenómenos escapan a nuestra observación.

Por eso me parece muy interesante referirme a los estudios y experimentos que llevaron a cabo Snyder y Rosen. feld desde el año 1936, respecto a la actividad del ástema respiratorio en la vida intranterina $y$ que están con. signados en la abra -Obstetric analge. sia and Anesthesia, de Snyder (1950).

En ellos quedó cláramenté establecido que así como el centro cardiaco bulbar del ioto es cactivo, lo mismo acurre con el centro respiratorio $y$ que de clerta época do la vida intrauteri- na en adelante, se establecen los movimientos respirátorios en el lelo; que en el caso del humano tienen un ritmo de 60 por minuto a menos.

- La experimentación en la especie numaina es dificil y complicada $\bar{y}$ no sería el caso exponerla aqui. No as: en la coneza donde es posible obser. var-directamente al teto a iraves de las paredes transparentes del utero en estado de gravidez, previa laparotomia.

- Estos inyestigadores estudiaron el efecto de casi todos los anestésicos inhalatorios e intravenosos sobre la ácfividad respiratoria fetal en distintas especies, inclusive la humana, y pudieron comprobar que todos los anestésicos que se suministran a la madre provocan en e! feto uña depresión o abolicion de los movimientos respiratorios. Fal cosa encontraron con el Nembutal venoso. Paraidehido, Cloral hidrato. Mortina, Eter. Etilleno y Protóxido de nitrógeno: la única excepción es al Ciclopropano que no produce ninguna depressión fetal, a pesar de estar la madre en anestesia profunda, de aqui su muy buena aplicación en to cesáreá

El Pentotal también produce como las demás drajas depresión respirátoria en el telo pero más aceñtuada, más patente cuin estando la mádre bastán. to despierta sin haber entrado en pla. no anestésico. Tiene da ventaía de su rapida-metabolización, de suerte que pasados unos pocos minutos después de-la inyección todo vuelve a lo nor. mal. De los barbitúricos el Pentotal es el que metaboliza con más rapidez.

Retiriéndonós chóra a nuestros ca. sos podemos estudiar las stonientes cosas: 
numas letales. I enienao e. cuiaado de auscultar con el estetoscopio después de cada dosis de Pentotal, se ob serva con frecuencia que los latidos cardíacos del feto se hacen más lentos muy pasajeramente. Es posible que haya una ligera depresión del centro cardíaco bulbar. Nosotros anotamos cuatro casos sin importancia alguna

Peso del niño. Todas las criaturas estaban a término o muy cerća de él. Los pesos osciloron entre 2.400 y 3.700 kilogramos. Solamente en un emba razo gemelar los fetos pesarón cada uno 1.800-gramos. No encontramos ninguna relación entre el peso y la anestesia.

Respiración. Se estableció inmediata. mente en 24 casos. En seis casos no fue inmediata sino más o menos tardia. Vale la pena que estudiemos un poco más detenidamente estos casos:

a) En dos niños (casos números 14 y 24), la respiración se demoró un minuto y $1 \frac{1}{2}$ minutos, respectivamente. pero luégo lloraron espontáneamente sin tener que recurtir ni a inyecciones ni a maniobras resucitadoras. Los par tos fueron normales, las dosis de Pen total no fueron altas, pero los niños nacieron un poco dormidos. Este retardo es seguramente debido al anestésico

b) En un caso (número 1), el niño nació dormido, con movimientos res piratorios superficiales y color azula do: se aplicó Lobelina $y$ se adminis. uó oxígeno a presión $y$-rodo entró en lo normal. El parto fue normal-y las dosis de Pentotal moderadas. Este es un caso de depresión respiratoria.

c) En los tres casos restantes (núme ros 17, 20 y 26), los niños nacleron sin respirar y cianosados, hubo que ha cerles maniobras resucitadoras y apli. carles oxigeno. Pero aqui no podemos atribuírle al Pentotal la culpa de es tos accidentes, pues en el caso núme so 17 se aplicó un fórceps por sufrimiento fetal y se comprobó un nudo del cordón que se había apretado; on el caso número 20 fue necesario aplicar un tórceps por trabajo prolongado de bido a una posicion O.I.I.P dellejada que no progresaba y el teto comenza ba a sutrir; en el caso número $26 \mathrm{se}$ presento una procidencia del cordón con gran suirimiento fetal y de urgen. cia se aplicó un lórceps. En este últímo caso es probable que por la compresión del cordón a hacerse prociden. te ni siquiera hubiera pasado Pento tal al feto.

En resumen, solamente en tres casos podemos culpor al Pentotal de la depresión respiratoria, que por lo de. más no revistió ninguna gravedad.

El doctor J. C. Whyte, del Ottwa Civic Hospital, publica en el número del mes de enero de 1952 del Ampri. can Journal of Obstetrics and Gynecology (página 163), un estudio sobre 824 partos de los cuạles 500 ariestesió con Pentotal venoso a $5 \%$ y 324 con otros agentes anestésicos, y encontró que de los niños nacidos baio aneste sia pentotálica el $15 \%$ necesitaron maniobras resucttadoras y que de los nacidos bajo otras anestesias, el $18 \%$ necesitaron de esas maniobras. Lo que quiere decir que la incidencia de depresiones respiratorias es sensibie. mente Igual con el Pentotal que con cualquier otro cinestésico.

Color del niño. Fue rosado al nacer en tödas las criaturas, con excepción de los seis anteriores casos que no respiraron inmediatamente y que na. cieron de un color más o menos azu. 
. Ose y cuyas causas ya se analizaron en el párrato anterior.

Meconio. A este punto le puse especial atención por haber oido comentz. rios que es muy alto el porcentaje de niños que nacen expulsondo méconio. de donde se deducía que el Pentotal oroducia sufrimiento tetal; : aún xis :a la trase que el meconio era la ru brica del Penthotal., Al estudiar nueztro cuadro no veo iustificada tal aser. ción.

En efecto, observamos el mecónio en seis niños pèro dentro de las siguientes circunstancias:

a) En tres niños habia previo sufrimiento fetal y todos requirieron extracción con fórceps. Son los mismos casos que vimos antes, a saber: un nuco del cordón que se crpretó, una posición O.I.I.P. deflejada sin progrescr a pesar de buenas contracciones, y una procidencia del cordón.

b) Hubo dos ntños que expulsaron ineconio, pero uno era una presenta - ción de nalgas en embararo único $y$ este fenómeno es casi normal en esta modalidad de presentación; el otro ocurrió en un segundo gemelo que venía en presentadón podálica.

c) Finalmente hubo uno que expulsó meconio sin causa manifiesta fcano número 18), $y$ en el cual tampocs estamos cutorizados para decir que el Pentotal es el responsable de tal he cho, pues no hay baves para jusgatio.

De suerte que la expulisión do meconio está ligada más estrechamenie al sufrimiento fetal por cansa obstétri. ca que al cmestésico empleado.

Headura del cardbn Usando ei Pentotal como anestbalco, debe ligarse el cordón prontamente para evitar que continue pasando la draga de la circulación materna a la fetal.

Curare. Como bien lo sabemos, el Curare tiene la propiedad de relajur los músculos estriados cuando se inyecta por via venosa o intramuscular (esta via es mucho márs tardia) y la re lajación es más a menos proporcio nal a la dosis empleada. Por su ac. ción simpaticolítica deja predominancia vagal y de esta manera viene u ser indirectamente tonificante de la fibra muscular uterina.

En el comercio se encuentran dos tipos de la droga que son: los curares naturales - Tucurin, D-Tubocurarina. etc.) y los curares sintéticos (Flarredil. Myánesin, etc.) Los curares naturales vienen dosificados a 20 unidades par centímetro cúbico o $0.0 p 3$ gramos par centímetro cúbico. Un contímetro cúbi. co de los unos se corresponde en cuanto a su etecto a 1 c. c. de los otros. Los curares sintéticos tienen la ven. toja de no tener el factor broncojemparmb́dico y el de su duración más corta. lo quie tos hace más manojables.

En mis casos usé como curare na tural el Tucurin, y como curare sintí. tico el Floxedil. Las dosis que siempre inyecté, y no necesité ninguna máyor, tueron dos c. c. por vía venosa tónto de los unos como de los otros, y me pare. cieron sus efectos símilares e ipuatmente buenos.

- En únlco fin con que los usé fue peire observar su poder de relatación solvire los músculos pertnectes contrcícos $\gamma$ wa beneficto en las maniobras obstbtricas. Pude observar que la acción del Curare es bastante buena $Y$ aprecicible como relaiadora del pertent, thento qu en muchos casos evtia las potelolo 
mías que no dudariamos un solo instante de practicar para evitar desgarros. En aplicaciones de fórceps es singuiarmente útil por la relajación que se consigue.

La técnica de su uso es muy simple. Como obra tan rápidamente; pues a Jos tres minutos ya se ven los efectos, basta con inyectarlo por vía ver:asa minutos antes del desprendimiento de la presentación, por la misma aquja del Pentotal y con la enferma dormda. La inyección debe hacerse lentamente observando la respiractón de la paciente, pues es un depresor de la función respíataria y su acción se suma a la del Pentotal. Por precaución siempre debe tenerse oxígeno a la mano y los elementos de intubación encotraqueal por si se presenta algim accidente.

Teniendo cuidado en el manejo del Curare son muy raras las complicaciones: en unos 200 curares que yo he aplicado en total para cmèstesias generales y usando dosis muchístmo más altas que en maternidad, nunca se me ha presentado ningún acciciente.

En los casos del presente estudio lo usé 11 veces, cinco con Tucurín $y$ seis con Flaxedil. Este último tiene la ventaja de poderse usar simultáneamente por la misma aguja con el Pentotal porque no se precipita en su presencia, cosa que no ocurre con el Tucuriñ qué si se precipita len este caso dobe canalizarse otra vena).

Analicemos nuestros casos en los cuajes se usó Curare y Pentotal. Debemos advertir que el Curare no pasa por la placenta de la madre al niño y en caso que así ocurra, la can. tided es tan pequefia que puede des. preciarse.
Los casos los-distribuímos enire cuatro primíparas y siete multíparas.

Primíparar. Dentro de éstas hubo dos áplicaciones de fórceps y un embarazo gemelar con segundo feto en presentación podálica. En el fórceps del caso número siete hubo un desgarro perineal de segundo grado por estrechez de los tejidos. En el caso nú. mero 20 de otro fórceps, en el embarazo gemelar $y$ en el otro parto normal hubo buena relajación y no hubo necesidad de episiotomia.

Multiparas. En las siete multíparas la relajación fue en todas muy buena y no se necesitó la episiotomía ni se presentaron desgarros. Dentro de éstas hubo presentación podálica en embarazo único, el Curare relajó satisfactoriamente y se facilitó mucho la extracción de la cabeza última.

En total; de los 11 casos, en 10 de ellos la acción del Curare fue excelente y evitó en muchas ocasiones las episiotomias. En un caso hubo desgarro de segundo grado por estrechez de los teilidos.

De estas pocas observaciones me ha quedado la ldea que donde mejor obran los curares es en las multíparas con perinés contracturados; allí se obtiene una amplia relajación. En camblo en las primiparas con mu. cha frecuencla se observan estreche ces de los telidos, de modo que ol Curare reiaja los músculos peró nada puede contra la estrechez anatómica; aquí resulta buena la asociación de Ia droga con la eplsiotomía no muy extensa para aprovechar las ventajas de cadr uno. En las aplicaciones de Iórceps, en las maniobras obstétricas y en la presentcaión de pelvis es dos- 
de más beneficios podemos obteren del Curare.

Cuando se usa la droga en la en. ferma aún no darmida pueden presen. tarse trastornos visuales, como oct rrió en el caso número 27, debido a parálisis de la acomodación ocular, que por lo demás no tiene importan. cia ninguna.

\section{ADSUMaN}

El Pentotal venoso es un buen ancilósicoo obstétrico. Debe uearee en embarmos a término, nunca en prematunos.

La dosis inicial fluctúa entre 0.15 y 0.40 gramos. La dosis de mantenimiento oscila entre 0.03 y 0.10 gramos. La dosis total llega de 0.50 a 0.70 gramos.

E Pentotal tiene Jas siguientes VENTAJAS:

a) Fácil de adminiatrar.

b) Precio muy redueldo. dos.

c) No necesita aparatos complica.

d) Provoca una anestesia muy agradable.

e) No produce vómitos y por consi. guiente no es absolutamente neceso. rio que el estómago de la paciente esté vacío.

i) La anestesia se consigue en po. Cos segundos, sin exclitación.

c) Decpoder répido y coradable.

h) A la enteried lo quada un buen recuerdo do su cinestesia.

DESVENTAJAS:

a) Do clerta dosis para curiba retarda la trecuentia de las contracciones uterinas.

b) Disminuye la cantidad de las mitumpre. c) Acorta la duración de las con. tracciones.

d) Es mayor la incidencia en cuan. to al uso de ocitócicos.

e) Provoca depresión respiratoria fetal.

En consecuencia el Pentotal tam. poco llena las condiciones del anesté. sico ideal en obstetricia. Aún está por - descubrirlo.

Curax. Es un gran onuxiliar on obs. tetricia par la relajación de los músculos perinecles que provoca. Está muy indicado en las maniabras abstétricas, en la aplicación de fórceps y en la presentación de pelvis.

\section{AnHocunth}

Ciralde Lopes Illonso. Tesis de la Facultad. Boootés.

Geovqua Caibes A. Tesis de la Facul. tod. Bogoté.

Deso D. Inepido. Conducción del par to con onemteja con Rentotal. T. ats. Bowotd.

Henco Pencita laph, 2.800 partos con Pentotal: Medellín.

Mortíne Mipul Tratado de Anesto tesia. Barcelona.

D. Leo-Creonhill. Principles and Practice of Obstétrics.

8nive. Obstetrics Analgesia and Anesthesia. 1050.

1. Iatedil L'inosthesie tacllites por

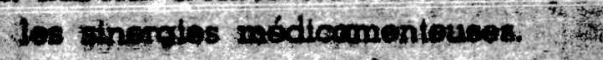
Decle. Documents expicimentaux of dinioues sur le Flarcall - 1852.

Epola Curare et Curarisants de Synthese.

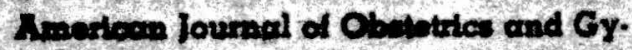
acodect. Enero de 1952.

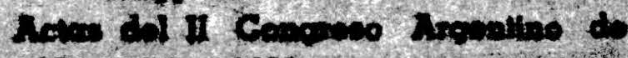

